\title{
On the mechanisms of icicle evolution
}

\author{
JEROME A. NEUFELD†, RAYMOND E. GOLDSTEIN \\ AND M. GRAE WORSTER
}

Institute of Theoretical Geophysics, Department of Applied Mathematics and Theoretical Physics, CMS, Wilberforce Road, Cambridge CB3 0WA, UK

(Received 4 September 2009; revised 8 December 2009; accepted 8 December 2009)

We present a study of a cylinder of ice melting in warm air in order to quantify the heat-transfer mechanisms controlling the evolution of its shape, which are inherent in a range of phenomena involving phase change and fluid flow. Motivated by the initial melting at the top of a flat-topped cylinder of ice, we analyse laminar, natural convection above a cooled, finite, horizontal plate (or below a heated, finite, horizontal plate) and show that, to a very good approximation, the partial-differential, boundary-layer equations can be separated with self-similar vertical profiles scaled by the boundary-layer thickness. We find that the horizontal evolution of the boundarylayer thickness is governed by equations describing a steady, viscous gravity current fed by diffusive entrainment, and therefore describe such flows as diffusive gravity currents. We first use the predictions of our model to examine previous experimental results in two dimensions. Our experimental results relating to the melting of ice in air are then compared with predictions based on our analysis of the axisymmetric thermal boundary layer. This comparison confirms the vertical thermal structure and shows that melting is governed in roughly equal measure by heat transfer from the air, the latent heat of condensation of water vapour, and the net radiative heat transfer from the surroundings to the ice.

\section{Introduction}

The natural environment is full of examples where phase change affects and is affected by fluid flows, from the intricate patterns of snowflakes to the solidified forms of lava eruptions and the sculpted shapes of stalactites. One intriguing example is the formation of icicles, during which melt water, for example from snow on a hot tin roof, freezes as it drips into cold air. The overall, elongated shape of an icicle, as well as ripples that form on it, have been thought to be a result of interactions between the thin film of melt water flowing on its surface and the solid ice beneath (Ogawa \& Furukawa 2002; Ueno 2003, 2004). Recently, Short, Baygents \& Goldstein (2006) suggested that the dominant heat transfer controlling the growth and form of icicles is not associated with the water film but rather with the convective boundary layer in the air surrounding them.

The growth of the tip of an icicle may be influenced by the drips that form there, and a cleaner way to investigate the interactions between convective airflow and phase change relevant to an icicle is to consider the melting of a cylinder of ice held vertically in warm air. An example of the melting of a right cylinder of ice is 

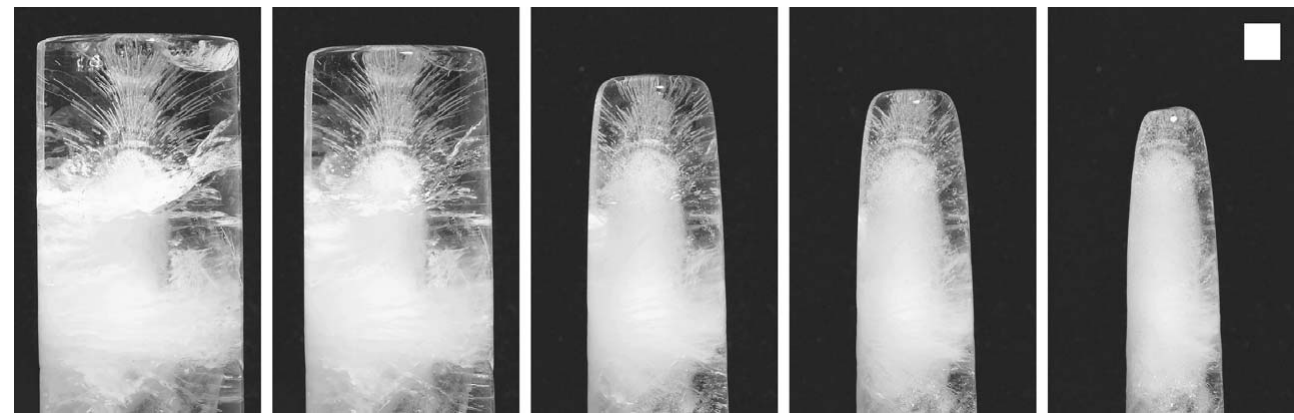

FIGURE 1. A sequence of high-resolution images showing the melting of a cylinder of ice in air of far-field temperature $T_{\infty}=21.4^{\circ} \mathrm{C}$ taken at times $t=10,51,114,164,214$ and $266 \mathrm{~min}$. The white square for scale is $1 \mathrm{~cm}$ on a side.

shown in figure 1 and movie 1 . The ice cools the surrounding air, which flows radially outwards along the top of the cylinder and down the curved sides. However, in what follows we focus our attention on the flow along the top surface of the cylinder of ice, highlighting the balance of physical effects needed to describe the form of the boundary layer. We leave for future discussion the ultimate evolution of the shape of the icicle.

Buoyancy-driven convection above a finite, horizontal, cooled plate without phase change (or equivalently below a heated plate) has been the subject of many previous studies, yet open questions remain. The cooled fluid (air) pours over the edges of the plate, and the flow is controlled by conditions at or near the edges, in the manner of a weir for example. There are excellent visualizations of such flows in the paper by Aihara, Yamada \& Endo (1972), and of the associated thermal boundary layer in the paper by Hatfield \& Edwards (1981). These papers, as well as our own experiments and numerical calculations, show the flow to be laminar and provide measurements of velocity and temperature profiles, as well as local and mean rates of heat transfer which we compare with our theoretical predictions.

Stewartson (1958) described the differential boundary-layer equations appropriate to such flows and found a similarity solution to them, which he incorrectly interpreted as the flow above a cooled, horizontal plate. His solution, which we summarize in $\S 2$, actually describes the flow from the leading edge above a heated, horizontal plate (Gill, Zeh \& Del Casal 1965).

Most analytical studies of convective flow adjacent to horizontal plates have used integral boundary-layer equations with assumed vertical shape functions introduced to evaluate the integrals. These are nicely summarized by Higuera (1993), who emphasizes the elliptic character of the problem and the need for a boundary condition at the edge of the plate. It had variously been proposed that the thickness of the boundary layer should be zero at the edge of the plate (Wagner 1956), or that it should attain a critical depth by analogy with open-channel hydraulics (Clifton \& Chapman 1969), or that a singularity in the integral equations should coincide with the edge (Singh \& Birkebak 1969), which was later shown to be equivalent to maximizing the heat flux from the plate (Fujii, Honda \& Morioka 1973). More recently, it has been suggested that the boundary layer should adjust so as to maximize the discharge rate (mass flux) over the edge (Dayan, Kushnir \& Ullmann 2002). Higuera (1993) himself put considerable effort into an analysis local to the edge of the plate and concluded that it seems likely that buoyancy in the corner region can deal with any flow supplied 
by the boundary layer, implying therefore that the mass flux reaching the edge must be as large as possible.

In this paper, we show that the partial differential equations governing flow and transport in the boundary layer can be separated into ordinary differential equations describing the vertical structures of the velocity, pressure and temperature fields and the horizontal variation of the boundary-layer thickness. The separation is exact at infinite Prandtl number, and we show it to be an excellent approximation for finite Prandtl numbers as low as that for air $(\approx 0.7)$. A similar approach has been used by Higuera \& Weidman (1995) to analyse the flow above a cooled plate in a porous medium. The equation governing the horizontal variation of boundary-layer thickness has the form of that describing a steady viscous gravity current in which the local divergence of fluid flux is balanced by diffusive entrainment, and the differential system is closed uniquely by requiring simply that the mass flux at the edge of the plate be non-zero and finite.

In $\S 2$, we present our theoretical approach in two dimensions and compare its predictions with existing experimental data and our own full numerical calculations. In $\S 3$, we extend our analysis to an axisymmetric disk and compare the predictions with measurements taken in air above a melting cylinder of ice. In $\S 4$, we apply our results to our measurements of the initial melting of a cylinder of ice and discover surprisingly large roles played by condensation of water vapour from the air and radiative heat transfer from the surroundings. General conclusions and possible future applications of this method of treating the boundary-layer structure are presented in $\S 5$.

\section{Convection above a two-dimensional strip}

\subsection{Theoretical development}

We consider buoyancy-driven flows above isothermal, horizontal plates, as illustrated in figure 2. The flow above a heated or cooled plate is exactly analogous to the flow beneath a cooled or heated plate, respectively, and our results can be applied to the latter cases by simply reversing the sign of gravity. When the plate is heated the fluid above forms a thin, laminar boundary layer that develops and grows as the fluid flows from the leading edge of the plate (see figure $2 a$ ). In contrast, when the surface is cooled the fluid above forms a thin, laminar boundary layer whose thickness diminishes as the fluid flows from the centre towards the trailing edge of the plate. In both cases, we consider laminar flow that can be well described by the usual Boussinesq boundary-layer equations when the distance along the plate is much larger than the characteristic boundary-layer thickness (Schlichting \& Gersten 2000).

The steady two-dimensional Boussinesq boundary-layer equations describing momentum balances and heat transfer above a horizontal plate are

$$
\begin{aligned}
u \frac{\partial u}{\partial x}+w \frac{\partial u}{\partial z} & =-\frac{1}{\rho_{\infty}} \frac{\partial P}{\partial x}+v \frac{\partial^{2} u}{\partial z^{2}}, \\
0 & =-\frac{1}{\rho_{\infty}} \frac{\partial P}{\partial z}-\frac{\rho-\rho_{\infty}}{\rho_{\infty}} g, \\
u \frac{\partial T}{\partial x}+w \frac{\partial T}{\partial z} & =\kappa \frac{\partial^{2} T}{\partial z^{2}},
\end{aligned}
$$



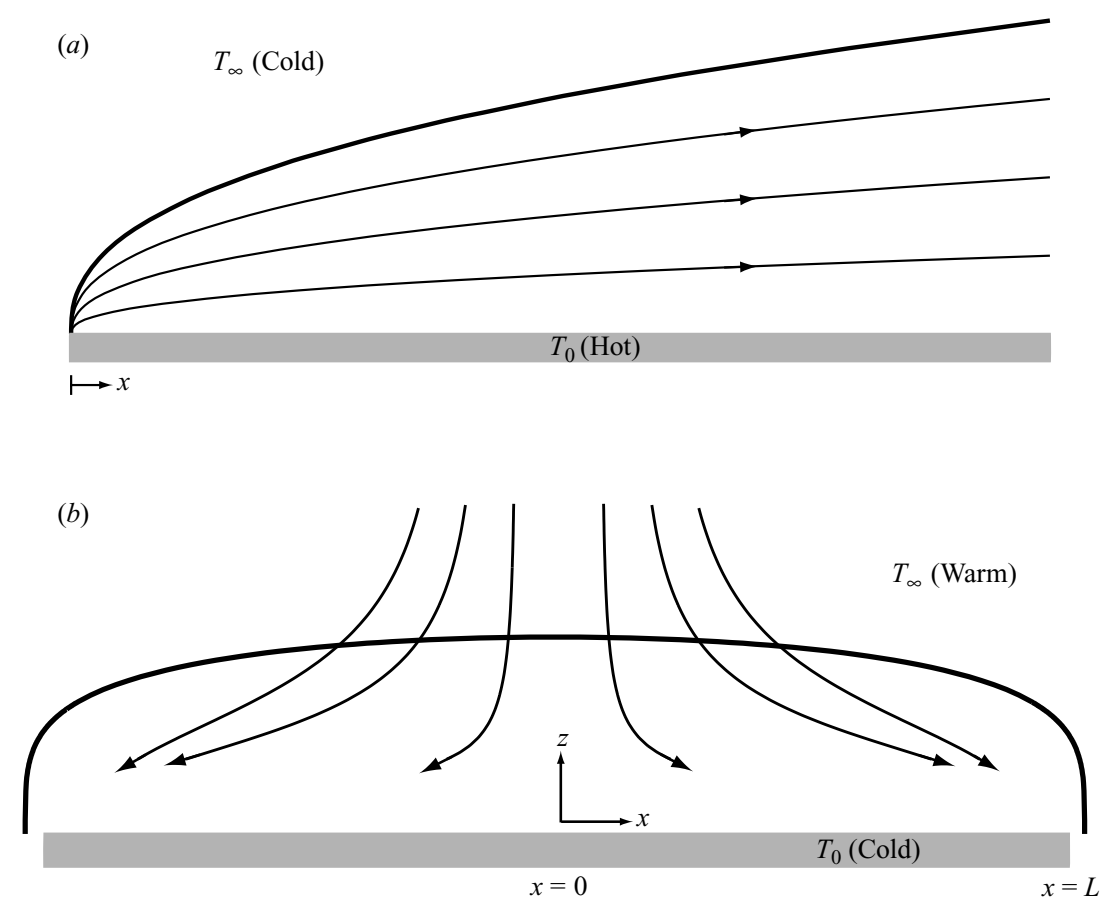

FIGURE 2. Geometry of convection above isothermal, horizontal plates. In (a) a warm thermal boundary-layer flows from the leading edge of a heated plate. The cool thermal boundary layer above a cold plate is shown in $(b)$. Near the centre of the plate, the flow has the character of a stagnation-point flow.

(Stewartson 1958). These are coupled by the linear equation of state

$$
\rho=\rho_{\infty}\left[1-\alpha\left(T-T_{\infty}\right)\right] .
$$

Continuity is assured by using a two-dimensional stream function $\psi(x, z)$ such that the velocity field $\boldsymbol{u}=(u, w)=\left(\psi_{z},-\psi_{x}\right)$. Here, $P(x, z)$ and $T(x, z)$ are the pressure and temperature fields, $x$ and $z$ are coordinates along and perpendicular to the plate, $v$ and $\kappa$ are the kinematic viscosity and thermal diffusivity of the fluid, $\alpha$ is the coefficient of thermal expansion, $\rho$ is the density field, and $\rho_{\infty}$ is a reference density of the fluid, measured at the far-field temperature $T_{\infty}$.

A number of previous authors (Gill et al. 1965; Wagner 1956; Singh \& Birkebak 1969 , for example) have approached this problem by assuming a prescribed vertical structure for the temperature, velocity and pressure fields. Here, we show that both the vertical structure and its lateral variation can be found independently by judicious approximation of (2.1) and (2.2). To that end we look for self-similar solutions of these equations of the form

$$
\begin{aligned}
& T(x, z)=T_{\infty}+\Delta T \theta(\eta), \\
& \psi(x, z)=\kappa q(x) f(\eta), \\
& P(x, z)=\rho_{\infty} \alpha \Delta T g h(x) p(\eta),
\end{aligned}
$$


where $\Delta T=\left|T_{0}-T_{\infty}\right|$ is the temperature difference driving fluid motion and $T_{0}$ is the temperature of the plate. The similarity variable

$$
\eta \equiv \frac{z}{h(x)}
$$

while the dimensionless functions $\theta(\eta), f(\eta)$ and $p(\eta)$ are vertical-structure functions for temperature, stream function and pressure respectively, and $q(x)$ and $h(x)$ represent the horizontal variations of the mass flux and the thickness of the boundary layer, respectively. Note that the spatial variables $x$ and $z$ and the thickness $h$ have so far been left dimensional. Substitution of these expressions into (2.1) and (2.2) leads to the set of differential equations

$$
\begin{aligned}
f^{\prime \prime \prime}+\sigma^{-1}\left[h q^{\prime} f f^{\prime \prime}+\left(q h^{\prime}-h q^{\prime}\right) f^{\prime 2}\right] & =\frac{\alpha g \Delta T}{\kappa \nu} \frac{h^{3} h^{\prime}}{q}\left(p-\eta p^{\prime}\right), \\
p^{\prime} & =\theta, \\
\theta^{\prime \prime}+h q^{\prime} f \theta^{\prime} & =0,
\end{aligned}
$$

where $\sigma=v / \kappa$ is the Prandtl number. These equations still involve two independent variables, $\eta$ and $x$, but the functions involved in them are all functions of just one independent variable. The primes denote differentiation with respect to the argument of the respective functions, either $\eta$ or $x$.

These equations can be made dimensionless by scaling $x$ with a horizontal scale $L$, such as the length of the plate, scaling $z$ and $h$ with $R a^{-1 / 5} L$ and scaling $q$ with $R a^{1 / 5}$, where the Rayleigh number

$$
R a=\frac{\alpha g \Delta T L^{3}}{\kappa \nu} .
$$

We see, therefore, that the boundary-layer assumption $h \ll x$ is appropriate provided the Rayleigh number $R a \gg 1$. In addition, these scalings provide a natural definition of the characteristic Reynolds number

$$
R e=\frac{u h}{v} \sim \frac{\kappa R a^{1 / 5}}{v}=\frac{R a^{1 / 5}}{\sigma} .
$$

For the experiments detailed in $\S \S 2$ and 4 the Reynolds number is $R e \simeq 10-35$, which is significantly less than the critical Reynolds number, $R e_{c r i t} \sim 400$, for instability of shear-driven flows (Drazin \& Reid 1981).

With these scalings, the governing equations

$$
\begin{aligned}
f^{\prime \prime \prime}+\sigma^{-1}\left[h q^{\prime} f f^{\prime \prime}+\left(q h^{\prime}-h q^{\prime}\right) f^{\prime 2}\right] & =\frac{h^{3} h^{\prime}}{q}\left(p-\eta p^{\prime}\right), \\
p^{\prime} & =\theta, \\
\theta^{\prime \prime}+h q^{\prime} f \theta^{\prime} & =0,
\end{aligned}
$$

are independent of Rayleigh number and depend only on the Prandtl number, so there is just a one-parameter family of solutions.

Our aim is to separate the vertical and horizontal structures of the boundary layer, resulting in decoupled sets of ordinary differential equations describing each. Equation (2.8b) already involves only the independent variable $\eta$. Equation $(2.8 c)$ and 
the viscous and buoyancy terms of $(2.8 a)$ can be separated by choosing

$$
h q^{\prime}=1 \quad \text { and } \quad \frac{h^{3} h^{\prime}}{q}= \pm 1
$$

Separation of $(2.8 c)$ requires that $h q^{\prime}$ be constant, and it is necessary for that constant to be positive in order that $\theta$ decays as $\eta \rightarrow \infty$. Without loss of generality, the constant can be set equal to unity in $(2.9 a)$. The right-hand side of $(2.8 a)$ must be negative in order that the vertical gradient of vorticity be negative near the plate, so $h^{3} h^{\prime} / q$ must be positive when $\theta$ is positive (the plate is heated) and negative when $\theta$ is negative (the plate is cooled). Again, without loss of generality, the magnitude of the constant in $(2.9 b)$ can be taken to be unity.

Once $(2.9 a-b)$ are satisfied, the equations describing the vertical structure $(2.8 a-c)$ become

$$
\begin{aligned}
f^{\prime \prime \prime}+\sigma^{-1}\left[f f^{\prime \prime}-\left(1-q h^{\prime}\right) f^{\prime 2}\right] & = \pm\left(p-\eta p^{\prime}\right), \\
p^{\prime} & =\theta, \\
\theta^{\prime \prime}+f \theta^{\prime} & =0 .
\end{aligned}
$$

These equations are subject to the boundary conditions

$$
\begin{aligned}
\theta= \pm 1, \quad f=f^{\prime}=0 & (\eta=0), \\
\theta \rightarrow 0, \quad p \rightarrow 0, \quad f^{\prime} \rightarrow 0 & (\eta \rightarrow \infty),
\end{aligned}
$$

which express the fact that the plate is isothermal and that the air is stationary there, and that in the far field, the temperature has a different uniform value, the pressure is known and the horizontal velocity vanishes.

We see that $(2.10 a)$ is not quite separated because $x$-dependence remains in the term $q h^{\prime}$. Below, we examine two cases in which the separation can be completed exactly: flow above a heated or cooled plate. We also show that, in the case of flow above a cooled plate, $q h^{\prime}$ is mostly very small, and that good approximate solutions can be obtained by either neglecting it or evaluating it locally.

\subsection{The heated plate}

It is clear that separation of $(2.10 a)$ is complete if $q h^{\prime}$ is constant. It is straightforward to show that for convection above a heated plate, as illustrated in figure $2(a)$, this condition with (2.9) leads to the power-law solutions

$$
h=\left(\frac{25}{6}\right)^{1 / 5} x^{2 / 5}, \quad q=\frac{2}{5}\left(\frac{25}{6}\right)^{4 / 5} x^{3 / 5},
$$

and that the momentum equation $(2.10 a)$ becomes

$$
f^{\prime \prime \prime}+\sigma^{-1}\left[f f^{\prime \prime}-\frac{1}{3} f^{\prime 2}\right]=p-\eta p^{\prime} .
$$

Note that the plus sign has been taken on the right-hand side, which is required for the flow above a heated plate, and that the plus sign must correspondingly be taken for the boundary condition on $\theta$ in (2.11a). Stewartson (1958) showed that such a power-law similarity solution does not exist when a minus sign is taken on the right-hand side of $(2.10 a)$, which is the case for flow above a cooled plate. The solution to $(2.13)$ with $(2.10 b),(2.10 c)$ and boundary conditions $(2.11)$ was presented by Stewartson (1958). 


\subsection{The cooled plate at infinite Prandtl number}

For either a cooled or a heated plate, it is straightforward to show that separation of the vertical and horizontal structure is complete in the limit $\sigma \rightarrow \infty$, when $(2.10 a)$ becomes

$$
f^{\prime \prime \prime}= \pm\left(p-\eta p^{\prime}\right)
$$

This equation describes the dynamics of the 'inner' thermal boundary layer, for which the appropriate far-field boundary condition on the velocity field is one of zero tangential stress, namely

$$
f^{\prime \prime} \rightarrow 0 \quad(\eta \rightarrow \infty)
$$

(see e.g. Kuiken 1968). The velocity relaxes to the far-field condition given by (2.11f) much farther from the plate in an 'outer' boundary layer that has a uniform temperature field and a balance between inertia and viscous dissipation. In the case of a cooled plate, illustrated in figure $2(b)$, solution of the governing equations for the thermal boundary layer shows that the dimensionless shear stress $f^{\prime \prime}(0)=1.378$, and the dimensionless temperature gradient $\theta^{\prime}(0)=0.607$.

The full, two-dimensional solution for the thermal boundary layer is completed by solving (2.9) for the horizontal variation of its thickness $h$ and volume flux $q$. Those equations can be written in the form

$$
-h^{3} h^{\prime}=q \quad \text { and } \quad q^{\prime}=h^{-1} .
$$

The first of these equations is recognizable as the relationship between the thickness and the volume flux in a two-dimensional, viscous gravity current. The second equation expresses a balance between the divergence of the horizontal volume flux, which gives the vertical entrainment velocity into the boundary layer, and the conductive heat flux across the boundary layer, which is inversely proportional to its thickness. This is the fundamental advection-diffusion balance that confines the boundary layer. We see here that the thermal boundary-layer flows exactly like a steady viscous gravity current whose thickness obeys the equation

$$
\left(h^{3} h^{\prime}\right)^{\prime}=-1 / h .
$$

The only difference is that the density field is smeared out by diffusion, according to $(2.10 c)$, rather than forming a discrete, two-layer system. We therefore call this a diffusive gravity current.

The boundary conditions for these equations on a finite plate are

$$
q=0 \quad(x=0) \quad \text { and } \quad q=q_{0} \quad(x=1),
$$

where the constant volume flux $q_{0}$ at the edge of the plate is finite. The first of these is the natural symmetry condition at the centre of the plate. The second is obviously true but would not seem to give sufficient additional information. However, we couple this statement with the observation that (2.17) is singular where $h=0$ and that the strength and form of the singularity can be chosen to ensure a finite volume flux. In other words, the flow over most of the plate 'sees' a finite sink near the edge of the plate. We expect that the location of the sink singularity should be within approximately one boundary-layer thickness of the edge of the plate so, within the same order of approximation as boundary-layer theory or lubrication theory, we place the singularity at the edge of the plate, where $x=1$. 

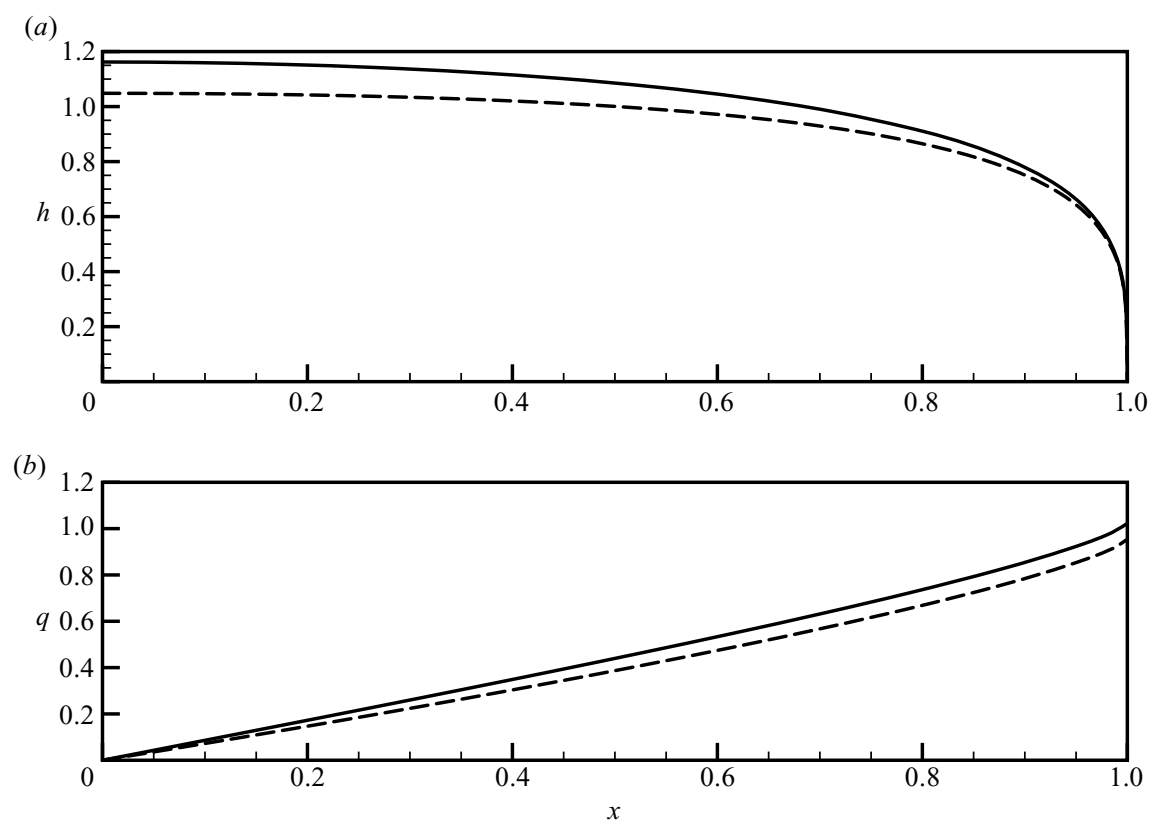

FIGURE 3. (a) Boundary-layer thickness and $(b)$ volume flux as functions of distance along the plate $x$. The solid curves show the solution to (2.16) with $q_{0}=1.023$ and $h(0)=1.162$. The dashed curves show the approximate solution (2.19), with $q_{0}=(64 / 81)^{1 / 5}$.

With these conditions, (2.16) can be expanded near $x=1$ to give

$$
h \sim\left(4 q_{0}\right)^{1 / 4}(1-x)^{1 / 4}-\left(3 q_{0}\right)^{-1}(1-x) \quad \text { and } \quad q \sim q_{0}-\frac{4}{3}\left(4 q_{0}\right)^{-1 / 4}(1-x)^{3 / 4} .(2.19 a, b)
$$

These asymptotic expressions can be used to initialize a relaxation scheme (we used Matlab's bvp4c routine) in which equations (2.16) are solved subject to condition (2.18a) at $x=0$ and the asymptotic expressions (2.19) at $x=1$. These three constraints provide the conditions necessary to determine the structure of the boundary layer and the flux $q_{0}$ at the edge of the plate $x=1$. The results are shown by the solid curves in figure 3 , for which $q_{0}=1.023$ and $h(0)=1.162$.

The asymptotic expressions near the nose can be used to provide approximate solutions over the whole domain by choosing $q_{0}=(64 / 81)^{1 / 5} \approx 0.954$ so that the value of $q$ given by (2.19b) is equal to zero at $x=0$. The result is shown by the dashed curves in figure 3 .

The thickness of the thermal boundary layer $h(x)$ and the resultant portrait of streamlines above the cooled plate are shown in figure 4 . The picture that emerges above the cold plate is of a steady, cold, viscous current fed by entrainment along its length as it propagates towards the edge of the plate.

The vertical structure is dominantly that of a thermal boundary layer in a stagnation point flow, for which the boundary-layer thickness is uniform (Worster 2000). The analysis of the gravity-current equation (2.17) including the singular edge condition is key to determining the strain rate of the stagnation point flow.

We can piece together the solutions above to find that the local dimensional heat flux from the plate

$$
F_{T}=-\left.k \frac{\partial T}{\partial z}\right|_{z=0}=-\frac{k \Delta T}{L} \operatorname{Ra}^{1 / 5} \frac{\theta^{\prime}(0)}{h(x)}
$$




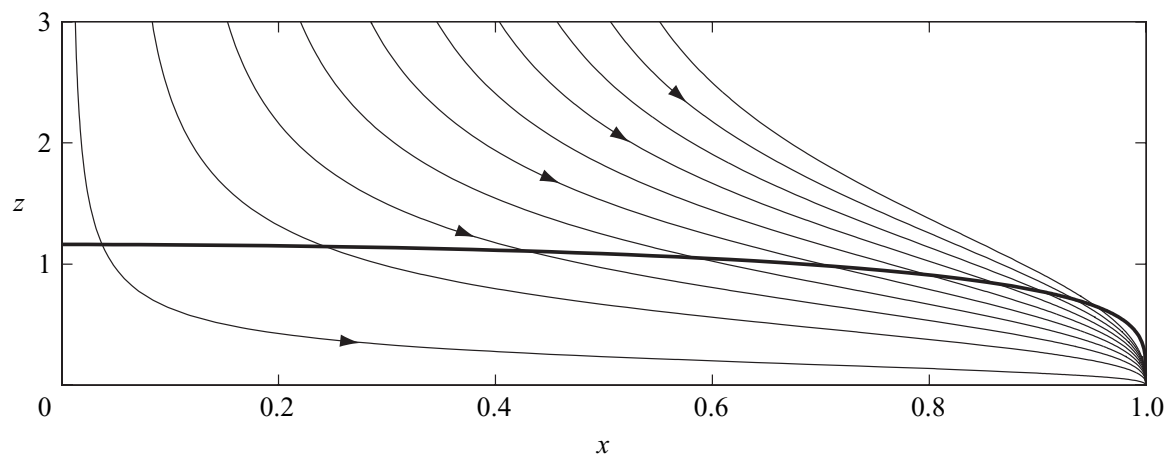

FiguRE 4. The thin curves show streamlines as functions of the horizontal and vertical coordinates $x$ and $z$, calculated at infinite Prandtl number. The bold curve indicates the boundary-layer thickness as a function of position along the plate and corresponds to an isotherm.

where $k$ is the thermal conductivity of the air. The non-dimensional heat flux towards the plate can be expressed in terms of a Nusselt number defined by

$$
N u \equiv \frac{F_{T}}{k \Delta T / L}=R a^{1 / 5} \frac{\theta^{\prime}(0)}{h(x)} .
$$

This measure of the local heat flux can be integrated to find the total heat flux towards the plate expressed in terms of a global Nusselt number

$$
\overline{N u}=R a^{1 / 5} \theta_{0}^{\prime} \int_{0}^{1} \frac{\mathrm{d} x}{h(x)} .
$$

In the limit of infinite Prandtl number, our solution gives

$$
\overline{N u} R a^{-1 / 5} \simeq 0.620 .
$$

This is shown by the dashed line in figure 6 . We see that it gives a good approximation (to within $10 \%$ ) of $\overline{N u}(\sigma) R a^{-1 / 5}$ down to Prandtl numbers of about 10 .

\subsection{Approximate results at finite Prandtl number}

When the Prandtl number is finite, effects of inertia begin to affect the thermal boundary layer. We see from $(2.10 a)$ that there are two inertial terms. The first term in square brackets, proportional to $f f^{\prime \prime}$, represents the vertical advection of vorticity and acts to increase the shear in the boundary layer and therefore to retard the flow. The second term, proportional to $f^{\prime 2}$, represents the divergence of the downstream momentum flux and can also be thought of as the kinetic energy that must be imparted to the fluid. This latter term has a coefficient that involves $q h^{\prime}$, which is the sole component frustrating complete separation of the boundary-layer equations at finite Prandtl number. However, $q$ and $h^{\prime}$ are both equal to zero at the centre of the plate, and figure 5 shows that the magnitude of $q h^{\prime}$ remains small for about $80 \%$ of the plate.

We can proceed to find approximate solutions either by setting $q h^{\prime}$ to zero or by treating $q h^{\prime}$ as a parameter given by the solutions to (2.16) above, and solving equations (2.10) subject to boundary conditions (2.11) at each downstream location $x$. The results obtained for the global Nusselt number from these two approaches are shown in figure 6 and differ by less than $10 \%$ for Prandtl numbers down to 0.5 . The 


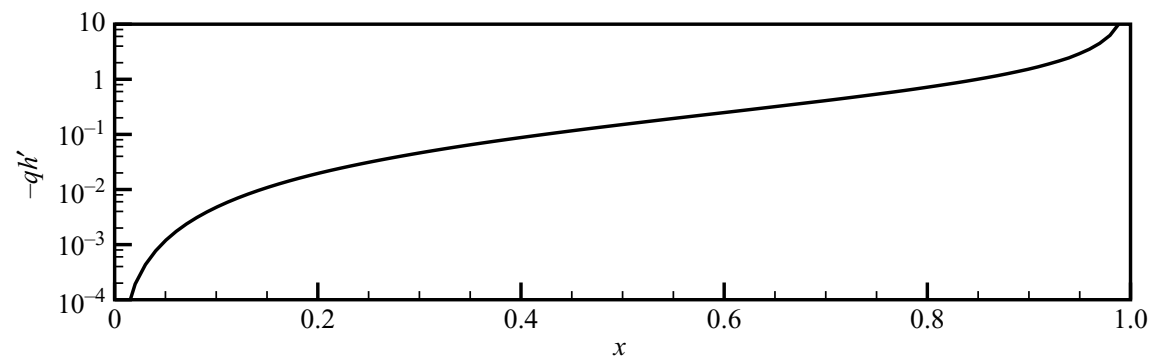

FIGURE 5. The magnitude of the residual inertial coefficient, $q h^{\prime}$, as a function of position along the plate.

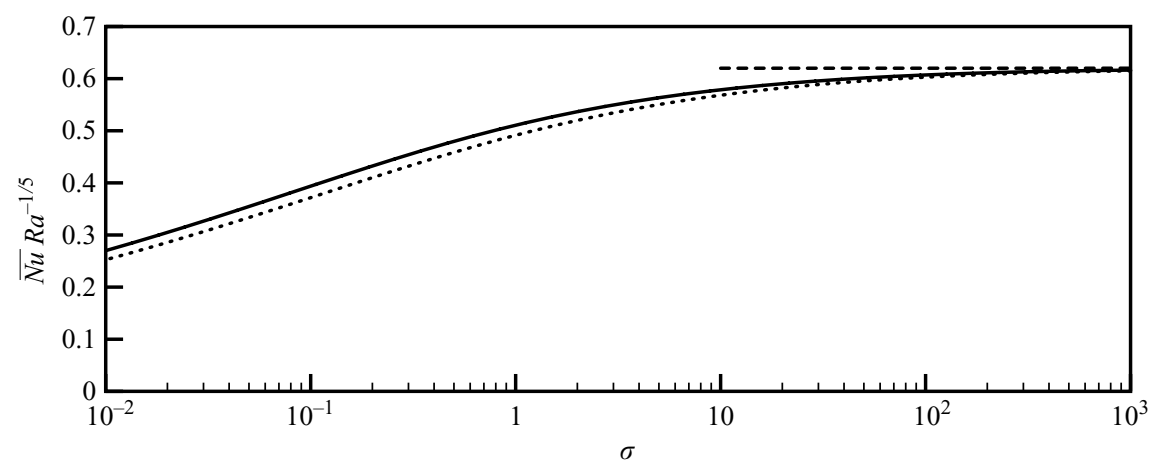

FIgURE 6. The solid curve shows the global Nusselt number $\overline{N u}$ scaled by $R a^{1 / 5}$, as a function of Prandtl number $\sigma$, computed by setting the residual inertial coefficient $q h^{\prime} \equiv 0$ in $(2.10 a)$. The dotted curve shows the solution obtained by evaluating the residual inertial coefficient locally. The heat flux in the limit $\sigma \rightarrow \infty$ is shown by the dashed line.

simplest approach is simply to neglect $q h^{\prime}$, so we do this henceforward in order to assess the utility of this approximation.

We compare our predictions with the results of Aihara et al. (1972), who conducted careful experiments in air below a heated plate $25 \mathrm{~cm}$ long. The experiments were confined by two glass plates, through which photographs were taken to record the trajectories of fine particles, from which measurements of the velocity field were made. In addition, vertical profiles of the temperature were recorded in various planes perpendicular to the plate using carefully suspended chromel-alumel thermocouples. Their data are shown in figures 7 and 8 alongside our theoretical predictions. We see that the velocity field is well predicted over most of the plate, though there are slight discrepancies near the edge, particularly in the outer part of the boundary layer. There seem to be slightly larger discrepancies in the structure of the temperature field. Importantly, however, the temperature gradient at the surface of the plate seems very well predicted. It should also be noted that no theory (ours included) predicts any negative velocities in the far-field $(z \rightarrow \infty)$ and that this aspect of the data may be primarily due to the return flow resulting from the necessity of doing experiments in a finite container.

In figure 9, we compare our results with the experimental measurements (Aihara et al. 1972) and our own numerical computations (see Appendix) of the local Nusselt number, scaled by the Rayleigh number, as a function of position along the plate, and find excellent agreement. We note that the local Nusselt number is constant along 


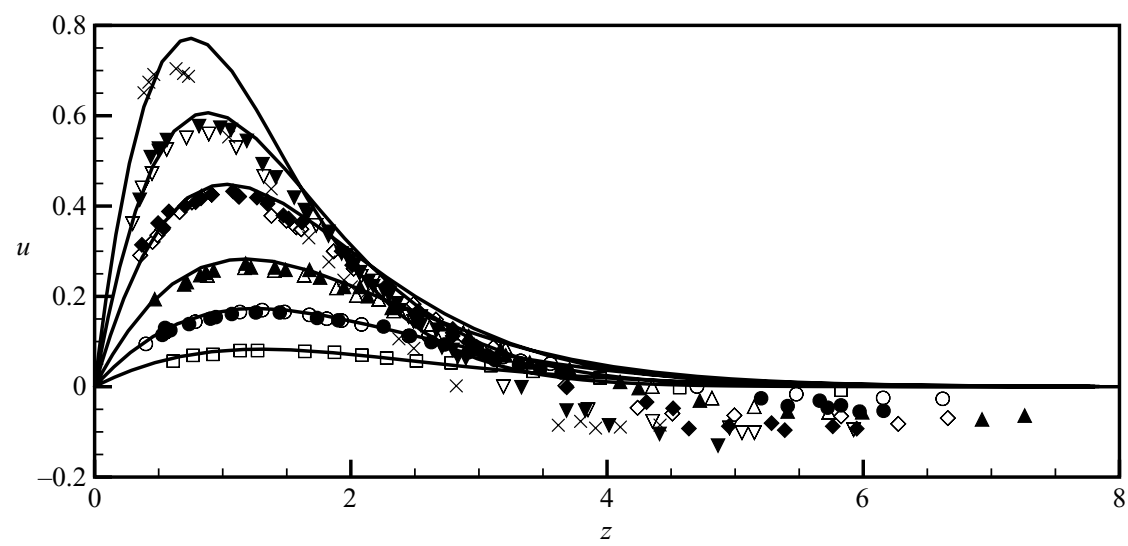

FIGURE 7. A comparison of the theoretical horizontal velocity structure (solid curves) and the experimental results of Aihara et al. (1972). Profiles are taken at $x=0.2$ (squares), $x=0.4$ (circles), $x=0.6$ (triangles), $x=0.8$ (diamonds), $x=0.9$ (inverted triangles) and $x=0.95$ (crosses). Open symbols correspond to a temperature difference of $\Delta T=55.2^{\circ} \mathrm{C}$ with an associated Rayleigh number of $R a=8.20 \times 10^{6}$. Solid symbols correspond to a temperature difference of $\Delta T=104.0^{\circ} \mathrm{C}$ and a Rayleigh number of $R a=1.17 \times 10^{7}$. We note that Aihara et al. (1972) ascribe a maximum error of $5 \%$ in the velocity measurements and discuss further in the text possible causes for $u(z>2)<0$.

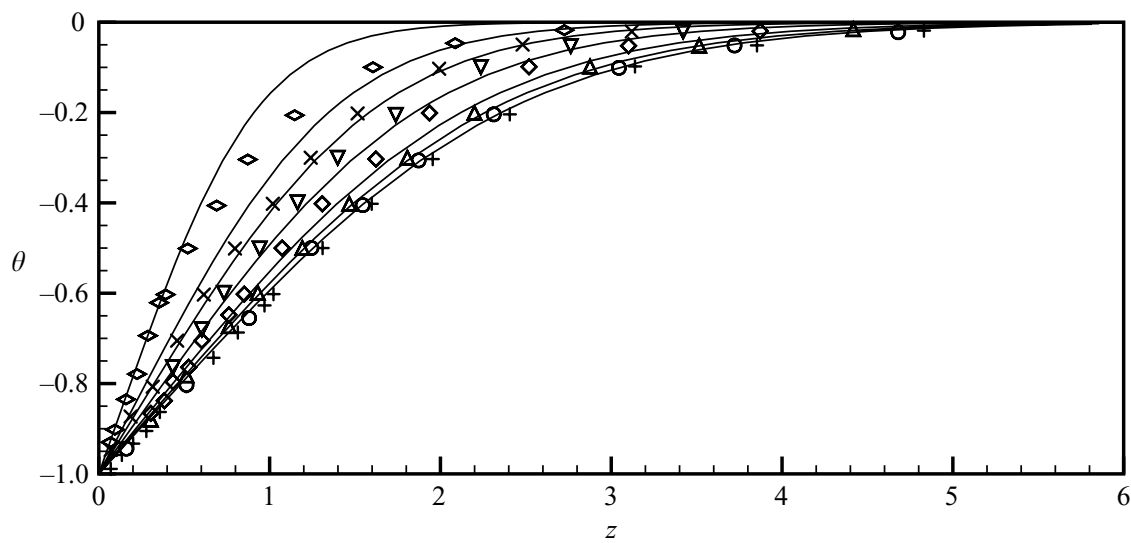

FIGURE 8. A comparison of the theoretical thermal structure (solid curves) and the experimental results of Aihara et al. (1972), for which $\Delta T=52.8^{\circ} \mathrm{C}$ and hence $R a=7.16 \times 10^{6}$. Profiles are from $x=0.0$ (plus), $x=0.4$ (circles), $x=0.6$ (triangles), $x=0.8$ (diamonds), $x=0.9$ (inverted triangles), $x=0.95$ (crosses) and $x=0.99$ (sideways diamonds). Experimental temperatures and distances are reported to have been measured to within $0.6 \%$ and $0.1 \mathrm{~mm}$, respectively (Aihara et al. 1972).

much of the plate, reflecting the structure found in the boundary-layer thickness $h(x)$. By integrating our results, we find the global Nusselt number $\overline{N u} R a^{-1 / 5} \approx 0.496$, which is within $3 \%$ of the experimental estimates of $\overline{N u} R a^{-1 / 5} \approx 0.509$ for $R a=1.02 \times 10^{7}$ and $\overline{N u} R a^{-1 / 5} \approx 0.500$ for $R a=7.16 \times 10^{6}$.

Such good agreement may well be fortuitous given uncertainties in the experimental measurements as well as our own approximations. For example, there are likely to be edge effects in the experiments, associated with the difficulty of maintaining an isothermal plate in the presence of large heat fluxes and with the finite size of 


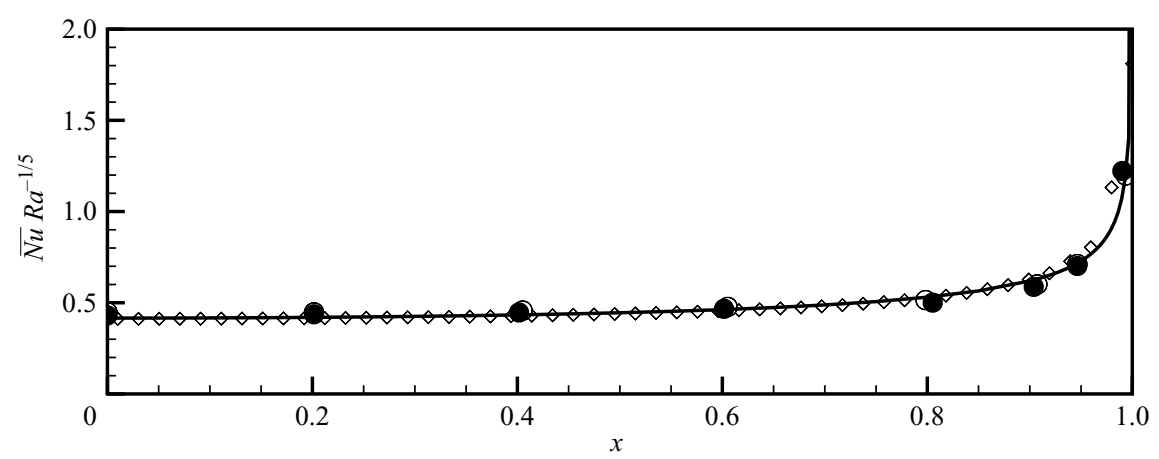

FIGURE 9. Local Nusselt number scaled by $R a^{-1 / 5}$ as a function of position along the plate for $\sigma=0.7$. The solid curve is the theoretical prediction made by ignoring the residual inertial coefficient. Results of the full numerical simulation for flow over a flat plate with $\sigma=0.7$ and $R a=2.83 \times 10^{6}$ are shown as diamonds (see the Appendix for full details of the calculation). Data from Aihara et al. (1972) are shown for two experiments in which $R a=7.16 \times 10^{6}$ (solid circles) and $R a=1.02 \times 10^{7}$ (open circles).

the bounding container, as well as edge effects in the theory associated with the breakdown of the boundary-layer hypothesis.

\section{Convection above a cooled circular disk}

The Boussinesq boundary-layer equations governing flow above an axisymmetric disk are identical to those for the flat plate (2.1)-(2.2), with $x$ replaced by the radial coordinate $r$. Continuity is now assured by introducing a Stokes stream function such that $\boldsymbol{u}=(u, w)=r^{-1}\left(\psi_{z},-\psi_{r}\right)$

Again, we look for self-similar solutions of the equations of the form

$$
\begin{aligned}
& T(r, z)=T_{\infty}+\Delta T \theta(\eta), \\
& \psi(r, z)=\kappa r q(r) f(\eta), \\
& P(r, z)=\rho_{\infty} \alpha \Delta T g h(r) p(\eta),
\end{aligned}
$$

where $\Delta T=\left|T_{0}-T_{\infty}\right|$ and the similarity variable $\eta=z / h(r)$. We scale $r$ by the radius of the disk $R$, the thickness of the boundary layer $h$ by $R a^{-1 / 5} R$ and the flux $q$ by $R a^{1 / 5}$, where now

$$
R a=\frac{\alpha g \Delta T R^{3}}{\kappa \nu}
$$

The dimensionless functions $\theta(\eta), f(\eta)$ and $p(\eta)$, specifying the vertical structure of the thermal field, the stream function and the pressure respectively, obey the unseparated differential equations

$$
\begin{gathered}
f^{\prime \prime \prime}+\sigma^{-1}\left[\frac{h}{r}(r q)^{\prime} f f^{\prime \prime}+\left(q h^{\prime}-h q^{\prime}\right) f^{\prime 2}\right]=\frac{h^{3} h^{\prime}}{q}\left(p-\eta p^{\prime}\right), \\
p^{\prime}=\theta \\
\theta^{\prime \prime}+\frac{h}{r}(r q)^{\prime} f \theta^{\prime}=0 .
\end{gathered}
$$




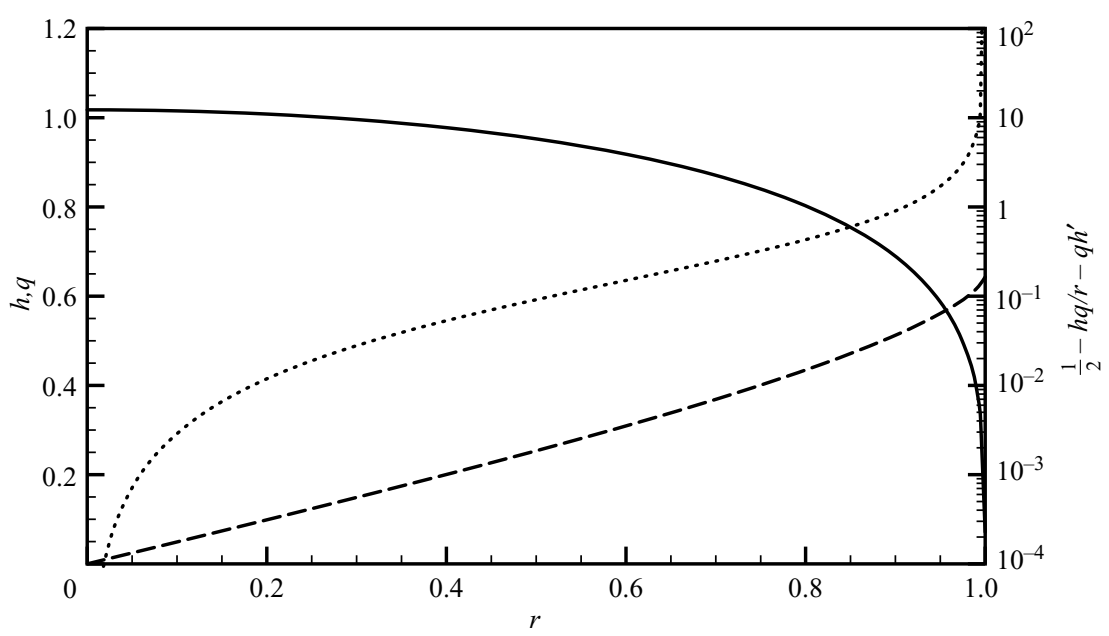

FIGURE 10. Radial boundary-layer profile $h(r)$ (solid), flux $q(r)$ (dashed) and the residual term $\frac{1}{2}-h q / r-q h^{\prime}$ (dotted) as functions of radial position.

Following the same procedure to separate these equations as we used in the twodimensional case, we choose

$$
\frac{h}{r}(r q)^{\prime}=1 \quad \text { and } \quad h^{3} h^{\prime}=-q .
$$

We identify the leading-order contribution to the horizontal inertia (proportional to $\left.f^{\prime 2}\right)$ by noting from $(3.4 a)$ that

$$
h q^{\prime} \sim \frac{h q}{r} \rightarrow \frac{1}{2} \quad \text { as } \quad r \rightarrow 0,
$$

and writing the equations governing the vertical structure in the form

$$
\begin{gathered}
f^{\prime \prime \prime}+\sigma^{-1}\left[f f^{\prime \prime}-\frac{1}{2} f^{\prime 2}+\left(q h^{\prime}+\frac{q h}{r}-\frac{1}{2}\right) f^{\prime 2}\right]=-\left(p-\eta p^{\prime}\right), \\
p^{\prime}=\theta, \\
\theta^{\prime \prime}+f \theta^{\prime}=0 .
\end{gathered}
$$

Note that the three (non-separated) terms enclosed in round brackets multiplying the inertial term $f^{\prime 2}$ sum to zero at $r=0$, and we find below that their sum remains small over most of the plate.

Equations (3.4) were solved numerically using the boundary conditions

$$
q=0 \quad(r=0) \quad \text { and } q=q_{0} \quad(r=1),
$$

and noting that the asymptotic form of $h(r)$ near $r=1$ is identical (to the order shown) to that given by $(2.19 a)$, with $x$ replaced by $r$. The resultant boundary-layer structure is shown in figure 10. We find the boundary-layer depth at the origin $h(0)=1.018$ and the flux at the edge of the disk $q_{0}=0.643$. These solutions are used to calculate the non-separated inertial coefficient (in the round brackets in $(3.6 a)$ ), which is shown by the dotted curve in figure 10 . We see again that this term is very small over most of the plate, and we therefore neglect it in our analysis for simplicity. 


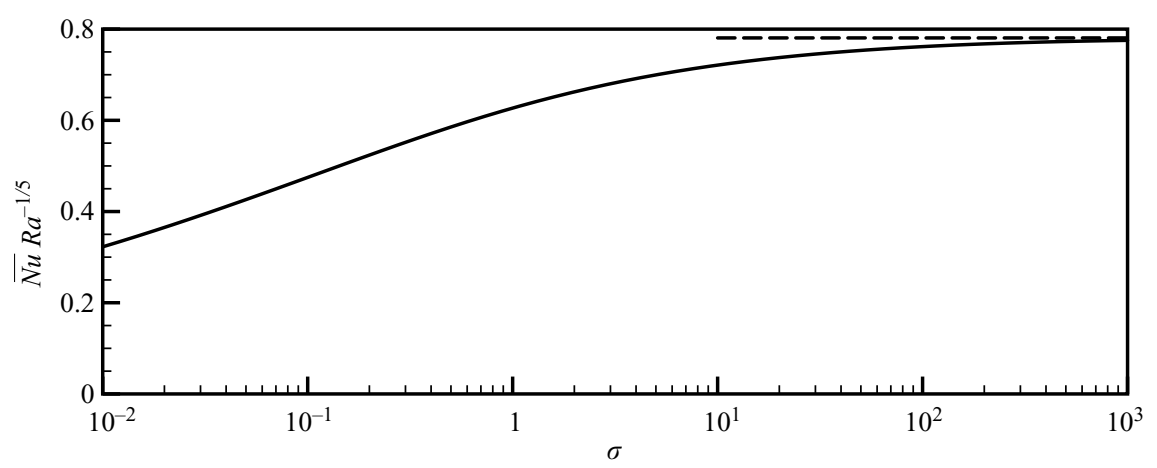

FIGURE 11. The solid curve shows the global Nusselt number scaled by the Rayleigh number, $\overline{N u} R a^{-1 / 5}$, as a function of Prandtl number $\sigma$. The limit $\sigma \rightarrow \infty$, in which the separation of horizontal and vertical structures is exact, is shown by the dashed line.

In the radial geometry, the local Nusselt number is defined by

$$
N u \equiv \frac{F_{T}}{k \Delta T / R}=R a^{1 / 5} \frac{\theta_{0}^{\prime}}{h(r)},
$$

where the local dimensional heat flux

$$
F_{T}=-\left.k \frac{\partial T}{\partial z}\right|_{z=0}
$$

The local Nusselt number determines the melt rate as a function of $r$ which is shown below in figure 13, where it is compared with results obtained from the experiment on the melting of a cylinder of ice described in $\S 4$.

A global Nusselt number can be defined as

$$
\overline{N u} \equiv \frac{\int_{0}^{R} N u 2 \pi r \mathrm{~d} r}{\pi R^{2}}=2 R a^{1 / 5} \theta^{\prime}(0) \int_{0}^{1} \frac{r \mathrm{~d} r}{h(r)} .
$$

We show the variation in the scaled global Nusselt number with Prandtl number in figure 11. For air at $0^{\circ} \mathrm{C}$, for which $\sigma=0.7$, we find that $\overline{N u} R a^{-1 / 5} \simeq 0.615$, a value which compares well with the experiments described below.

\section{The melting of ice}

Our study of convective boundary layers above cooled, horizontal surfaces is motivated by the shapes of icicles and spurred by the experimental results described below. We started with a block of cylindrical ice with a flat, horizontal top (see figure 1). These ice cylinders were placed in an enclosure at room temperature to protect them from air currents. Digital images were acquired every $15 \mathrm{~s}$ with a $4 \times 4 \mathrm{~kb}$ high-resolution Hasselblad camera. Illumination was provided by a flash unit triggered simultaneously with the camera to minimize heating from the light source.

Using a pair of $6^{\prime \prime}, \mathrm{f} / 4$ parabolic mirrors, we also acquired a series of Schlieren images, from which a measure of the thermal field in the air above the icicles could be derived. The intensity $I(x, z)$ of a Schlieren image is proportional to the integral of vertical temperature gradients along ray paths. Inverting the image to reconstruct 


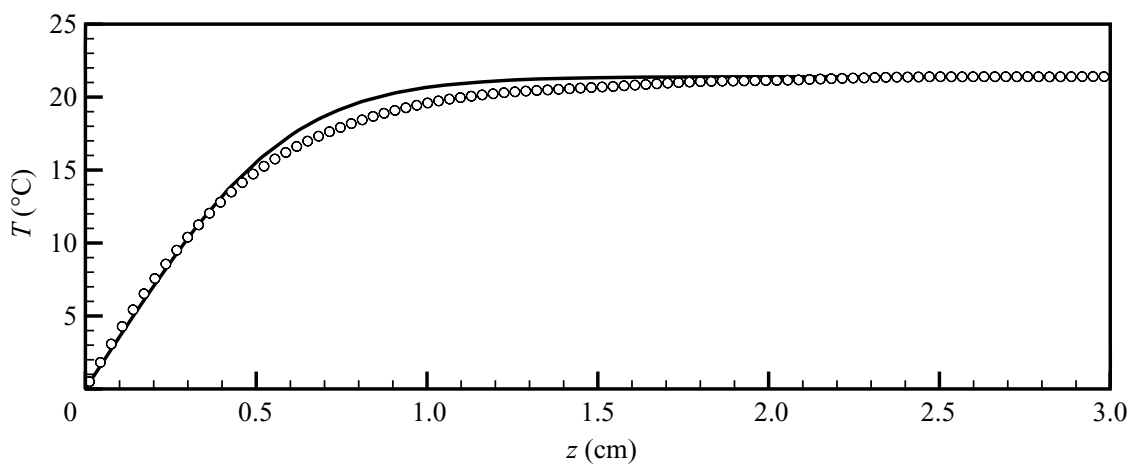

FiguRE 12. The thermal profile above an icicle of radius $R=2.7 \mathrm{~cm}$, as measured by the Schlieren apparatus (circles), is compared with the predicted thermal profile at the centre of the disk (solid curve) using the parameters given in tables 1 and 2.

the axisymmetric temperature field is a complex inverse problem. However, given that the boundary layer has a roughly constant thickness, we can estimate the temperature profile at the centre of the disk $T_{c}(z)$ by

$$
T_{c}(z)=T_{\infty}+\left(T_{0}-T_{\infty}\right) \frac{\int_{z}^{\infty} I\left(z^{\prime}\right)-I_{\infty} \mathrm{d} z^{\prime}}{\int_{0}^{\infty} I\left(z^{\prime}\right)-I_{\infty} \mathrm{d} z^{\prime}},
$$

where $I_{\infty}$ is the intensity of the background. The result is shown in figure 12, where we see that the data fit the theoretical prediction for the shape (in particular the boundary-layer thickness) of the thermal profile reasonably well. This gives us some confidence that our theory gives the correct rate of heat transfer from the air when we try to predict the melt rate of the ice below.

The optical images were analysed using a thresholding procedure to produce measurements of the position of the surface of the ice as it evolved in time. From these profiles we measured the rate of melting $v(r)$ along the top of the ice. The local melt rate at the top of the block of ice can be quantitatively explained in terms of three heat-transfer processes as detailed below: heat transfer from the air, the latent heat of condensation of water vapour and the net radiative heat transfer from the surroundings.

\subsection{Heat transfer from the air}

Conservation of heat at the ice-air interface is described by the Stefan condition

$$
\rho_{s} \mathscr{L} v=\left.k \frac{\partial T}{\partial z}\right|_{z=0},
$$

where $\rho_{s}$ is the density of ice, and $\mathscr{L}$ is the latent heat of melting per unit mass. This equation can be rearranged and evaluated using the theoretical model from $\S 3$ to obtain the melt rate at the centre of the top surface

$$
v=\frac{\rho}{\rho_{s}} \frac{c_{p} \Delta T}{\mathscr{L}} \frac{\kappa}{R} R a^{1 / 5} \frac{\theta^{\prime}(0)}{h_{0}},
$$

recalling that $k=\rho c_{p} \kappa$, where $c_{p}$ is the specific heat capacity of the air. 


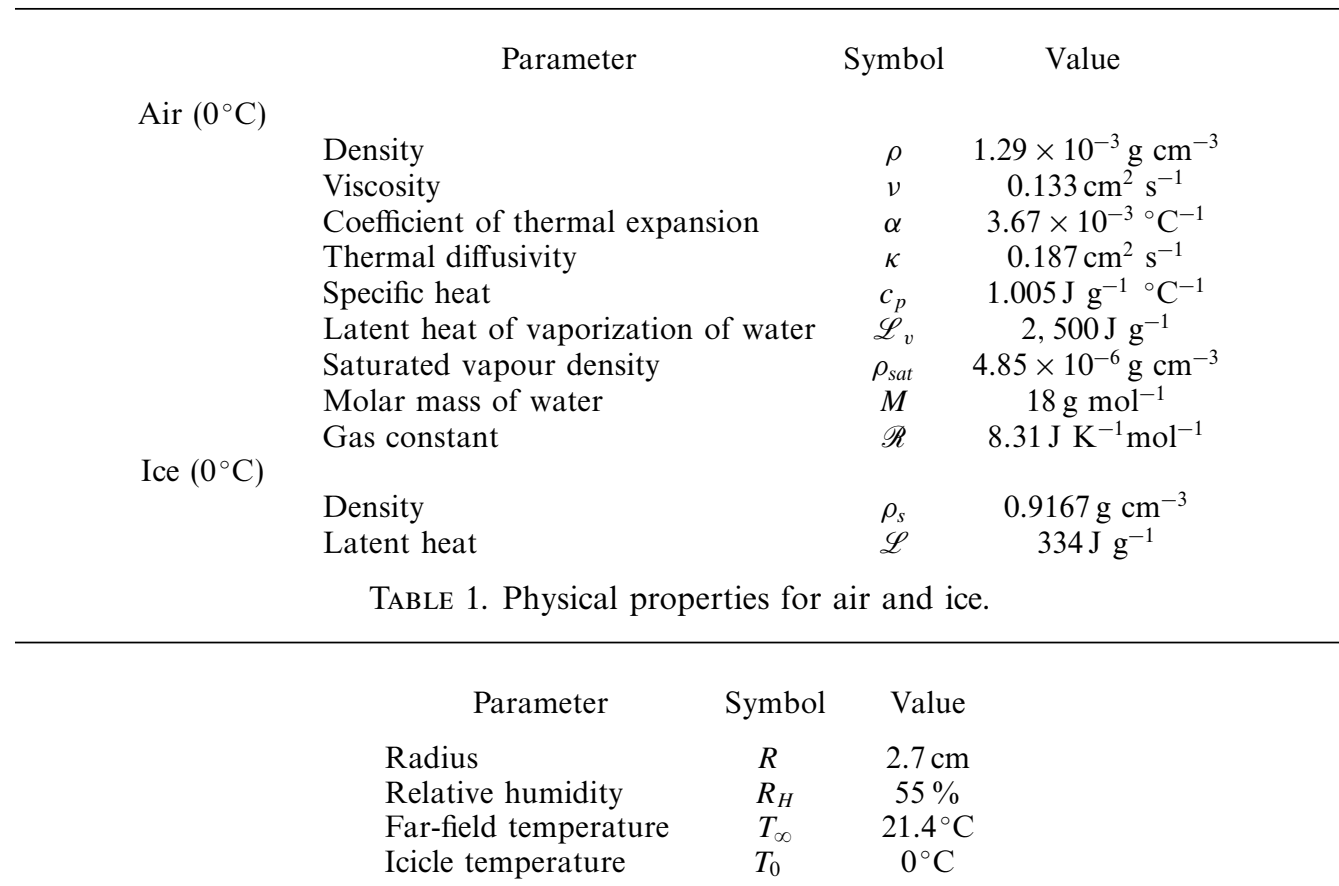

TABle 2. Parameters specific to the ice analysed in the experiment detailed in the text.

Using the physical parameters given in table 1 and the experimental parameters given in table 2, in which we have assumed that the surface of the ice is isothermal at the melting temperature $0^{\circ} \mathrm{C}$, we calculate the Rayleigh number $R a \approx 61000$ and the melt rate $v \approx 5.59 \times 10^{-5} \theta^{\prime}(0) \mathrm{cm} \mathrm{s}^{-1}$. At a Prandtl number of $\sigma=0.7$, our solutions give $\theta^{\prime}(0) \approx 0.483$, which gives a melt rate $v \approx 2.70 \times 10^{-5} \mathrm{~cm} \mathrm{~s}^{-1}$. This prediction contrasts with our measurement of $v \approx 7.9 \pm 0.1 \times 10^{-5} \mathrm{~cm} \mathrm{~s}^{-1}$, which indicates that the thermal boundary layer is not solely responsible for the observed melting.

\subsection{The meltwater film}

Many previous theoretical studies of the growth of icicles have focused attention on the thin film of water coating their surfaces. In the present context, it is straightforward to take account of the film within the same self-similar framework used in $\S 3$ to evaluate the thermal boundary layer in the air. Thin-film (lubrication) theory shows that the thickness $h_{w}$ of the meltwater film satisfies

$$
\rho_{w} \frac{g}{3 v_{w}} \frac{1}{r} \frac{\partial}{\partial r}\left(r h_{w}^{3} \frac{\partial h_{w}}{\partial r}\right)=\rho_{s} v,
$$

where $\rho_{w}$ and $v_{w}$ are the density and kinematic viscosity of water. This equation can be combined with (4.3) to show that $h_{w}$ satisfies exactly the same form of equation as $h$ and therefore, given also that they satisfy the same boundary conditions, that $h_{w}=\lambda h$, where

$$
\lambda^{4}=3 \alpha \Delta T \frac{\rho}{\rho_{w}} \frac{v_{w}}{v} \frac{c_{p} \Delta T}{\mathscr{L}} \theta^{\prime}(0) .
$$

The parameter values in tables 1 and 2 give $\lambda \approx 0.025$, so the meltwater film has a thickness of about $100 \mu \mathrm{m}$, compared with the thermal boundary-layer thickness of 
about $6 \mathrm{~mm}$. The balance of heat flux across the film requires that

$$
k_{w} \frac{\Delta T_{w}}{h_{w}} \sim k \frac{\Delta T}{h},
$$

which shows that the temperature difference across the film $\Delta T_{w}$ is less than approximately one hundredth of a degree (Short 2006). The meltwater film is therefore entirely negligible and, in particular, cannot account for the discrepancy between observed and predicted melt rates.

\subsection{Condensation of water vapour}

Having estimated and eliminated several other hypotheses for this discrepancy, we came to wonder about the role of water vapour in the air, recalling the adage, "It's not the heat that'll kill you, it's the humidity!" The water vapour has little influence on the thermal conductivity of the air at the temperatures involved in our experiments (Tsilingiris 2008). However, the airflow resulting from thermal convection carries water vapour to the surface of the ice, where it condenses and releases latent heat. The water vapour can be treated as a passive scalar because it has negligible influence on the density of air compared with temperature (see below). The vapour density satisfies $(2.1 c)$ with the thermal diffusivity $\kappa$ replaced by the diffusivity of water vapour $D$. It is readily shown by scaling that the gradient of vapour density is therefore equal to $\left(\Delta \rho_{v} / \Delta T\right)(\kappa / D)$ times the thermal gradient, and that the flux of water from the air to the ice surface is therefore

$$
F_{w}=\kappa \frac{\Delta \rho_{v}}{R} R a^{1 / 5} \frac{\theta^{\prime}(0)}{h},
$$

independent of $D$, where $\Delta \rho_{v}$ is the difference in the partial density of water vapour between the far field and the ice surface.

The saturated partial water-vapour density is given as a function of absolute temperature $T$ by

$$
\rho_{\text {sat }}(T)=\rho_{\text {sat }}\left(T_{0}\right) \frac{T_{0}}{T} \exp \left[-\frac{M \mathscr{L}_{v}}{\mathscr{R}}\left(\frac{1}{T}-\frac{1}{T_{0}}\right)\right],
$$

(Wood \& Battino 1990) where $T_{0}$ is a reference temperature (taken here to be the freezing temperature $T_{0}=273 \mathrm{~K}$ ), $M$ is the molar mass of water, $\mathscr{L}_{v}$ is the latent heat of vaporization, and $\mathscr{R}$ is the gas constant. We assume that the air is saturated at the ice surface, in which case

$$
\Delta \rho_{v}=R_{H} \rho_{\text {sat }}\left(T_{\infty}\right)-\rho_{\text {sat }}\left(T_{0}\right),
$$

where $R_{H}$ is the relative humidity of the air in the laboratory. Given the values in tables 1 and $2, \Delta \rho_{v} \approx 1.09 \times 10^{-5} \mathrm{~g} \mathrm{~cm}^{-3}$. This is about 100 times smaller than the density difference $\alpha \Delta T$ associated with temperature variations in the air, so it is appropriate to treat the water vapour as a passive scalar.

The Stefan condition is modified by the additional latent heat associated with condensation of water vapour to become

$$
\rho_{s} \mathscr{L} v=\left.k \frac{\partial T}{\partial z}\right|_{z=0}+\mathscr{L}_{v} F_{w},
$$

which can be rearranged to determine the melt rate

$$
v=\frac{\rho}{\rho_{s}} \frac{c_{p} \Delta T}{\mathscr{L}} \frac{\kappa}{R}\left[1+\frac{\Delta \rho_{v}}{\rho} \frac{\mathscr{L}_{v}}{c_{p} \Delta T}\right] R a^{1 / 5} \frac{\theta^{\prime}(0)}{h_{0}} .
$$




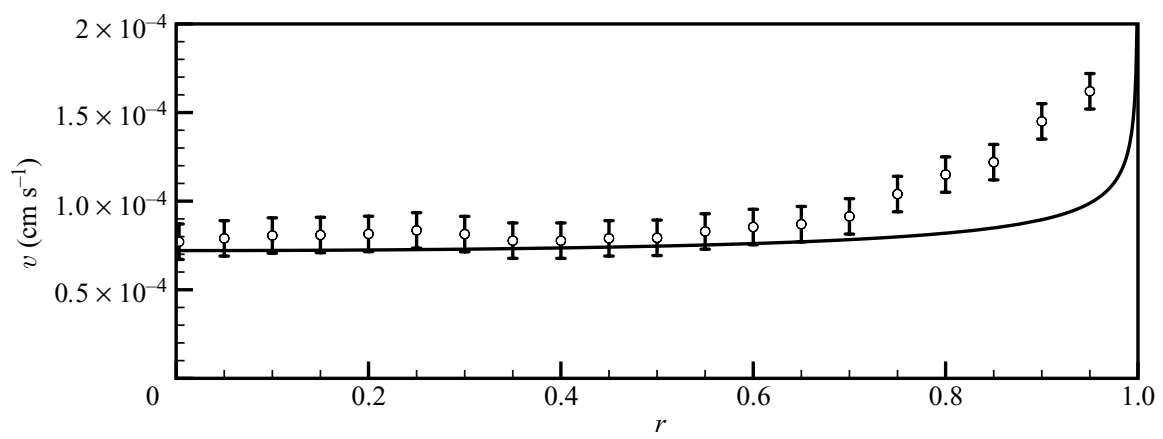

FIgURE 13. The predicted (solid curve) and measured (circles with error bars) melt rate as functions of radial position along the top of an icicle.

The second term in the square brackets has a value close to unity, which shows that the latent heat associated with condensation of water vapour contributes roughly the same amount of heat as is supplied by conduction. Our new prediction for the melt rate based on (4.11) is $v \approx 4.06 \times 10^{-5} \mathrm{~cm} \mathrm{~s}^{-1}$, which is still only about $55 \%$ of the measured value.

\subsection{Radiative heat flux}

The final piece of the jigsaw comes from a realization that the radiative heat transfer plays a role of similar magnitude to the sensible and latent heat fluxes. Conservation of heat at the ice-air interface is therefore given by

$$
\rho_{s} \mathscr{L} v=\left.k \frac{\partial T}{\partial z}\right|_{z=0}+\mathscr{L}_{v} F_{w}+F_{R}
$$

where the radiative flux

$$
F_{R}=\sigma_{B}\left(T_{\infty}^{4}-T_{0}^{4}\right),
$$

in which $\sigma_{B}$ is the Stefan-Boltzmann constant, and the temperatures must be expressed in Kelvin. This estimate of the radiation flux is based on assuming that the experimental surroundings radiate as a black body. Ice itself is almost a black body in the infra-red spectrum: it has a reflectivity of only about $1 \%$ and an 'optical' depth around $10 \mu \mathrm{m}$ at wavelengths around $10 \mu \mathrm{m}$ (Warren 1984), which is the peak of the black-body spectrum at the room temperature of $295 \mathrm{~K}$. Water has similar optical properties to ice in these conditions, so any water film present may also serve to absorb incoming radiation. Therefore, nearly all the incident radiation is absorbed at or near the ice surface, where it contributes to melting.

The melt rate at the centre of the ice based on (4.12) is $v \approx 7.71 \times 10^{-5} \mathrm{~cm} \mathrm{~s}^{-1}$, which is within $3 \%$ of the measured value of $v \approx 7.9 \pm 0.1 \times 10^{-5} \mathrm{~cm} \mathrm{~s}^{-1}$ and therefore well within the accuracy of our experiment.

The measured and computed vertical melt rates are shown as functions of radial position in figure 13. Note that the calculations assume a perfectly horizontal top surface, whereas the ice quickly gains a slightly rounded surface. Once that happens, the component of gravity parallel to the flow should accelerate the air, creating additional horizontal divergence and an associated thinning of the boundary layer and enhancement of the heat transfer. This effect is likely to be most important near the rounded edge of the ice and may account for the discrepancy in figure 13 for 
$r>0.7$. Prediction of the subsequent evolution of the shape of icicles awaits future evaluation.

\section{Conclusions}

In this paper, we have revisited the natural, buoyancy-driven flow above a cooled, finite, horizontal plate, which is equivalent to the flow below a heated plate. We have shown that, to a very good approximation, the thermal boundary-layer equations can be satisfied with self-similar solutions in which the horizontal variation of the scale height of the boundary layer obeys the thin-film (lubrication) equations for a viscous gravity current. These solutions make predictions that are in excellent agreement with previous experimental measurements and with a numerical simulation based on the full Navier-Stokes equations. However, while this validates the approach, our solutions are not significantly more accurate than previous approximations made using integral approaches. The importance of our work lies rather in the identification of the relationship between the boundary-layer equations and those governing viscous gravity currents, and the simplicity of the approach: the solution consists in solving one system of ordinary differential equations for the self-similar vertical structure of the boundary layer and another ordinary differential equation for the horizontal variation. This approach might prove useful in analysing other types of diffusive gravity currents.

A particular feature of our approach is the recognition that, in the case of a horizontal plate, the interior of the boundary layer sees the edge of the plate, where the dense fluid spills over, as a point sink whose strength is uniquely determined by the upstream conditions. It is likely therefore that the various conditions that have been proposed at the edge of the plate only affect conditions locally and do not have a leading-order effect on the global heat transfer.

We have applied our analysis of the thermal boundary layer to the air flow above a cylinder of ice placed in warm, still air in order to calculate the corresponding initial rate of melting, while the ice block has a horizontal top surface. We have shown that heat transfer from the air is insufficient to account for the observed rate of melting but that both the latent heat of condensation of water vapour and the net radiative heat flux from the surroundings to the ice must be accounted for. For typical icicles in air, these three mechanisms provide roughly equal contributions to the melt rate.

We have shown that the film of meltwater is entirely negligible. Given its very narrow width and high thermal conductivity relative to the thermal boundary layer in the air, it is almost isothermal and the heat flux across it is determined by heat transfer in the air. This can be deduced by scaling analysis and we speculate here that the film of water on a growing icicle is similarly negligible except that, in the case of a growing icicle, it must exist in order for the icicle to continue to grow: a distinction can be made between icicles that are dripping, which therefore have a continuous film of water covering them and whose length can therefore increase, and those for which the film freezes before reaching the tip and simply fatten. An intriguing feature of growing icicles is that their surfaces become rippled, which is thought to be a consequence of some form of morphological instability. The results of this paper suggest that analyses of such instabilities should incorporate the convection in the air, the condensation or evaporation of water vapour, and radiation, one or more of which might account for the observed features, and should perhaps disregard the water film as a key component of the system. 
(a)
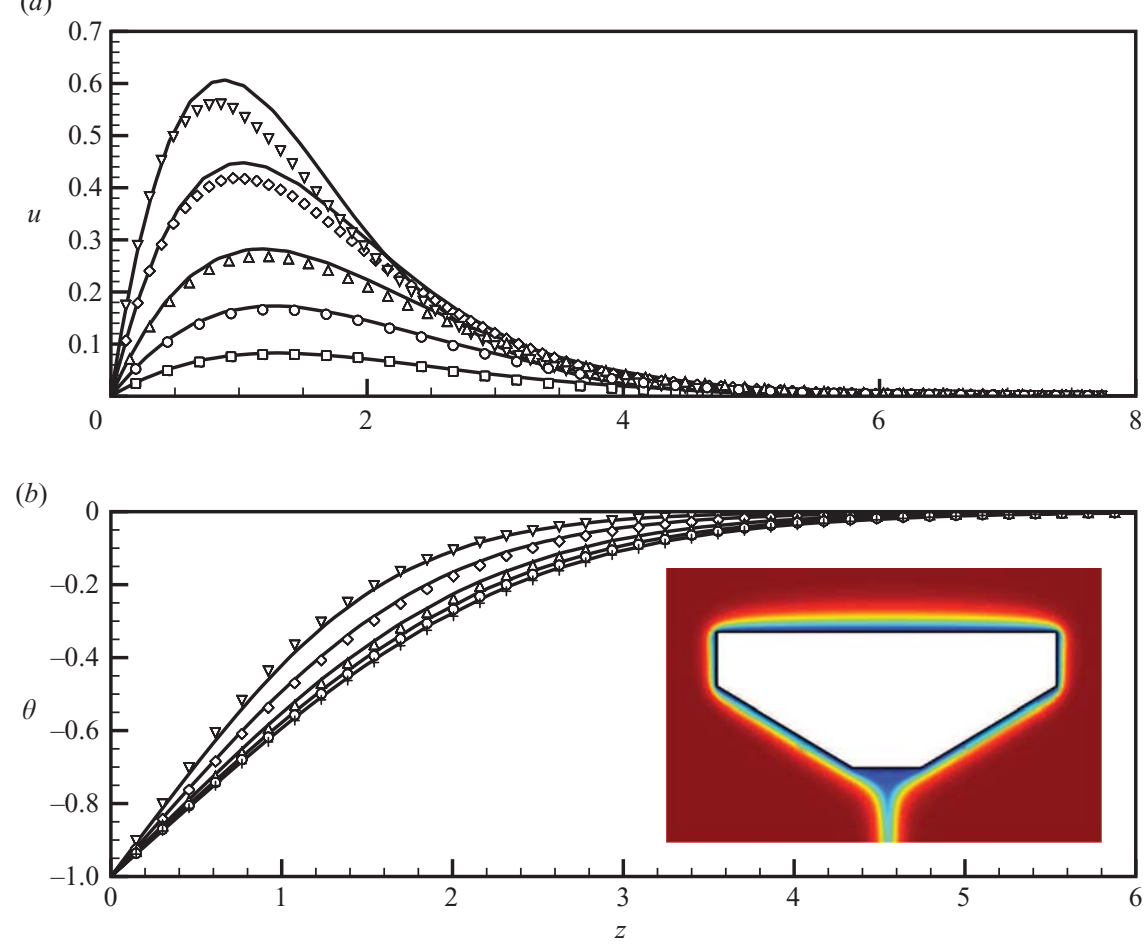

FIGURE 14. Numerical simulations of the full Navier-Stokes equations are compared with the theoretical predictions outlined in $\S 2$. Vertical profiles of the velocity are shown in $(a)$ at horizontal positions $x=0.2,0.4,0.6,0.8$ and 0.9 . The thermal profiles are shown in $(b)$ at horizontal positions $x=0,0.4,0.6,0.8,0.9$. The inset shows the thermal field around the blunt wedge with length (centre to edge) $L=12.5 \mathrm{~cm}$. The temperature difference is $\Delta T=10^{\circ} \mathrm{C}$; parameters representative of air were used, thus $\sigma=0.7$ and $R a=2.83 \times 10^{6}$.

We are very grateful to Rob Style for discussions about condensation. This work was supported in part by the Schlumberger Chair Fund. J. A. N. is supported by fellowships from Lloyd's Tercentenary Foundation and the Leverhulme Trust. M. G. W. was privileged to be a colleague of Steve Davis for three years in the Department of Engineering Sciences and Applied Mathematics, Northwestern University. At that time, around 1990, Steve ran two brown-bag-lunch seminar series, one on thin film flows and the other on solidification, which were wonderfully stimulating. We are pleased to offer this paper, combining aspects of both of those interests, in honour of Steve's 70th birthday.

\section{Appendix. Numerical solution of full Navier-Stokes equations}

As a complement to the boundary-layer approximation developed in the body of the present work, we have performed direct numerical simulations of the Boussinesq equations of motion for the thermal and velocity fields around a solid body held at fixed temperature. The numerical calculations were done with a commercial finite-element code (Comsol) which allowed for a non-uniform grid near the corners of the body. The upper surface of the body was chosen to have the shape of a rectangular slab, the bottom of which was deformed into a downward-facing blunted point in order to shed the descending boundary layer in a smooth manner (see the inset in figure 14b). 
All computations started with uniform plate and far-field temperatures and were conducted with $\Delta T=10^{\circ} \mathrm{C}$. Parameters representative of the experiments of Aihara et al. (1972) were chosen, namely $\alpha=3.67 \times 10^{-3}{ }^{\circ} \mathrm{C}^{-1}, \kappa=0.187 \mathrm{~cm}^{2} \mathrm{~s}^{-1}$, $v=0.133 \mathrm{~cm}^{2} \mathrm{~s}^{-1}$ and $L=12.5 \mathrm{~cm}$, which correspond to governing non-dimensional parameters $\sigma=0.7$ and $R a=2.83 \times 10^{6}$. The thermal boundary-layer structure was resolved with approximately 10 elements and was examined once the system had reached a steady state, characterized by the transport time across the plate. The resultant comparison between the numerically calculated velocity and thermal structure shown in figure 14 shows excellent agreement over most of the plate with the boundary-layer model of $\S 2$. Indeed, the excellent comparison between the boundary-layer model, experimental results and full numerical calculations lends credence to both the boundary-layer approximations and the explicit separation of vertical and horizontal structure. Thus, we find that the contribution of the thermal boundary layer to the melting of ice as exemplified by the variation of the local Nusselt number shown in figure 9 is well characterized by the boundary-layer method developed throughout this paper.

\section{REFERENCES}

Ainara, T., Yamada, Y. \& Endo, S. 1972 Free convection along the downward-facing surface of a heated horizontal plate. Intl J. Heat Mass Trans. 15, 2535-2549.

Clifton, J. V. \& Chapman, A. J. 1969 Natural-convection on a finite-size horizontal plate. Intl J. Heat Mass Trans. 12, 1573-1584.

Dayan, A., Kushnir, R. \& Ullmann, A. 2002 Laminar free convection underneath a hot horizontal infinite flat strip. Intl J. Heat Mass Trans. 45, 4021-4031.

Drazin, P. G. \& ReID, W. H. 1981 Hydrodynamic Stability. Cambridge University Press.

FujII, T., Honda, H. \& Morioka, I. 1973 A theoretical study of natural convection heat transfer from downward-facing horizontal surfaces with uniform heat flux. Intl J. Heat Mass Trans. 16, 611-627.

Gill, W. N., Zeh, D. W. \& Del Casal, E. 1965 Free convection on a horizontal plate. Zeit. Ang. Math. Phys. 16, 539-541.

HATFIELD, D. W. \& EDWARDS, D. K. 1981 Edge and aspect ratio effects on natural convection from the horizontal heated plate facing downwards. Intl J. Heat Mass Trans. 24 (6), 10191024.

Higuera, F. J. 1993 Natural convection below a downward facing horizontal plate. Eur. J. Mech. B. Fluids 12 (3), 289-311.

Higuera, F. J. \& Weidman, P. D. 1995 Natural-convection beneath a downward facing heated plate in a porous-medium. Eur. J. Mech. B. Fluids 14 (1), 29-40.

Kuiken, H. K. 1968 An asymptotic solution for large Prandtl number free convection. J. Engng Math. 2 (2), 355-371.

Ogawa, N. \& Furukawa, Y. 2002 Surface instability of icicles. Phys. Rev. E 66, 041202.

Schlichting, H. \& Gersten, K. 2000 Boundary Layer Theory. Springer.

Short, M. B., Baygents, J. C. \& Goldstein, R. E. 2006 A free-boundary theory for the shape of the ideal dripping icicle. Phy. Fluids 18, 083101.

Singh, S. N. \& BirkebAK, R. C. 1969 Laminar free convection from a horizontal infinite strip facing downwards. Zeit. Ang. Math. Phys. 20 (4), 454-461.

Stewartson, K. 1958 On the free convection from a horizontal plate. Zeit. Ang. Math. Phys. 9 (3), 276-282.

TsILINGIRIs, P. T. 2008 Thermophysical and transport properties of humid air at temperature range between 0 and $100^{\circ}$ C. Energy Convers. Manage. 49, 1098-1110.

Ueno, K. 2003 Pattern formation in crystal growth under parabolic shear flow. Phys. Rev. E 68, 021603.

Ueno, K. 2004 Pattern formation in crystal growth under parabolic shear flow. Part 2. Phys. Rev. E 69, 051604. 
WAGNER, C. 1956 Discussion of integral methods in natural convection flows by S. Levy. J. Appl. Mech. 78, 320-321.

Warren, S. G. 1984 Optical constants of ice from the ultraviolet to the microwave. Appl. Optics 23 (8), 1206-1225.

Wood, S. E. \& Battino, R. 1990 Thermodynamics of Chemical Systems. Cambridge University Press.

Worster, M. G. 2000 Solidification of fluids. In Perspectives in Fluid Dynamics: A Collective Introduction to Current Research (ed. G. K. Batchelor, H. K. Moffatt \& M. G. Worster), pp. 393-446. Cambridge University Press. 